\section{Giorgio Zanchin \\ Filippo Dainese \\ Federico Mainardi \\ Edoardo Mampreso \\ Chiara Perin \\ Ferdinando Maggioni}

\title{
Osmophobia in primary headaches
}

Published online: 20 July 2005

G. Zanchin $(\varangle) \cdot$ F. Dainese $・$ F. Mainardi

E. Mampreso • C. Perin • F. Maggioni

Headache Centre,

Department of Neurosciences,

University of Padua, Padua, Italy

e-mail: giorgio.zanchin@unipd.it

Tel.: +39-049-8211702

Fax: +39-049-8272335

\begin{abstract}
This study evaluates osmophobia (defined as an unpleasant perception, during a headache attack, of odours that are non-aversive or even pleasurable outside the attacks) in connection with the diagnosis of primary headaches. We recruited 775 patients from our Headache Centre (566 females, 209 males; age $38 \pm 12$ years), of whom 477 were migraineurs without aura (MO), 92 with aura (MA), 135 had episodic tension-type headache (ETTH), 44 episodic cluster headache (ECH), 2 chronic paroxysmal hemicrania (CPH) and 25 other primary headaches (OPHs: 12 primary stabbing headaches, 2 primary cough headaches, 3 primary exertional headaches, 2 primary headaches associated with sexual activity, 3 hypnic headaches, 2 primary thunderclap
\end{abstract}

headaches and 1 hemicrania continua). Among them, $43 \%$ with MO (205/477), 39\% with MA (36/92), and $7 \%$ with $\mathrm{CH}(3 / 44)$ reported osmophobia during the attacks; none of the 135 ETTH and $25 \mathrm{OPH}$ patients suffered this symptom. We conclude that osmophobia is a very specific marker to discriminate adequately between migraine (MO and MA) and ETTH; moreover, from this limited series it seems to be a good discriminant also for OPHs, and for $\mathrm{CH}$ patients not sharing neurovegetative symptoms with migraine. Therefore, osmophobia should be considered a good candidate as a new criterion for the diagnosis of migraine.

Key words Osmophobia $•$ Migraine Primary headaches $\cdot$ International Headache Classification

\section{Introduction}

In the new International Headache Society (IHS) classification [1], the alternative criteria for the diagnosis of migraine without aura that are reported in the appendix differ from the original criteria only in point $\mathrm{D}$, which requires the presence of at least two of the following: nau- sea, vomiting, photophobia, phonophobia and osmophobia. According to the Diagnostic and Therapeutic Guidelines of the Italian Society for the Study of Headaches [2], the presence of osmophobia is reported in the additional clinical information in favour of the diagnosis of headache; moreover, the Guidelines of the Canadian Headache Society [3] recommend in their criteria for the diagnosis of migraine the presence of osmo- 
phobia, which is judged to be highly sensitive and specific for migraine.

Intolerance to smell is often reported by migraine patients; despite this, the relationship between osmophobia and headaches has not been investigated in depth. Only two studies have evaluated the presence of osmophobia in migraine attack. The first, prior to the formulation of the IHS criteria [4], demonstrated it in $40 \%$ of 50 migraineurs studied [5]; the second, in a more recent study in a larger patient population, revealed the presence of osmophobia in $25 \%$ of migraineurs [6]. In a recent epidemiologic study of a Latin American patient population, osmophobia in migraineurs was said to be "almost always" present in $47.7 \%$ of subjects [7]. No study has ever been conducted that considered this phenomenon in relation to the different forms of primary headache.

\section{Subjects and methods}

We conducted a clinical study on a randomised sample of headache patients referred to our Headache Centre. The patients suffering from migraine were divided into those without (MO) and those with (MA) aura; others suffered from episodic tensiontype headache (ETTH), cluster headache and other trigeminal autonomic cephalalgias (TACs), and other primary headaches (OPHs). The diagnosis was formulated on the basis of the diag- nostic criteria of the 2004 IHS classification [1] following a history performed with a semi-structured questionnaire, general physical and neurological examinations, and, if needed, the exclusion of a secondary cause of headache by laboratory and/or diagnostic tests. Study exclusion criteria were concomitant migraine and ETTH, or other headaches, in the same subject; also excluded were patients with a diagnosis of probable primary headache.

A semi-structured questionnaire was administered to all patients to evaluate the eventual presence of osmophobia during a headache attack.

\section{Results}

A total of 775 patients (566 females, 209 males; age $38 \pm 12$ years) were recruited from our Headache Centre, of whom (Table 1) 477 had MO, 92 MA, 135 ETTH, 44 episodic cluster headache (ECH), 2 chronic paroxysmal hemicrania $(\mathrm{CPH}), 25$ other primary headaches (OPHs: 12 primary stabbing headaches, 2 primary cough headaches, 3 primary exertional headaches, 2 primary headaches associated with sexual activity, 3 hypnic headaches, 2 primary thunderclap headaches and 1 hemicrania continua). Among them, 43\% with MO (205/477), 39\% with MA (36/92) and 7\% with $\mathrm{CH}$ (3/44) reported osmophobia during the attacks; none among the 135 ETTH and $25 \mathrm{OPH}$ patients suffered this symptom (Table 2).

Table 1 Study population

\begin{tabular}{|c|c|c|c|c|}
\hline Diagnosis & $n$ & Age, years & $\mathrm{F}$ & M \\
\hline Migraine without aura (MO) & 477 & $38 \pm 12$ & 379 & 98 \\
\hline Migraine with aura (MA) & 92 & $37 \pm 11$ & 21 & 92 \\
\hline Episodic tension-type headache (ETTH) & 135 & $37 \pm 12$ & 95 & 40 \\
\hline Episodic cluster headache $(\mathrm{ECH})$ & 44 & $39 \pm 12$ & 7 & 37 \\
\hline Chronic paroxysmal hemicrania $(\mathrm{CPH})$ & 2 & $72 \pm 3$ & 2 & 0 \\
\hline Primary stabbing headache & 12 & $38 \pm 18$ & 8 & 4 \\
\hline Primary cough headache & 2 & $50 \pm 28$ & 0 & 2 \\
\hline Primary exertional headache & 3 & $43 \pm 23$ & 1 & 2 \\
\hline Primary headache associated with sexual activity & 2 & $37 \pm 10$ & 1 & 1 \\
\hline Hypnic headache & 3 & $63 \pm 11$ & 0 & 3 \\
\hline Primary thunderclap headache & 2 & $38 \pm 11$ & 1 & 1 \\
\hline Hemicrania continua & 1 & 40 & 1 & 0 \\
\hline Total & 775 & $38 \pm 12$ & 566 & 209 \\
\hline
\end{tabular}

Table 2 Patients who referred osmophobia $(n)$ during an attack

\begin{tabular}{lrrr}
\hline Diagnosis & $n$ & Total study population & $\%$ \\
\hline MO+MA & 241 & 569 & 42 \\
ETTH & 0 & 135 & 0 \\
ECH+CPH & 3 & 46 & 7 \\
OPHs & 0 & 25 & 0
\end{tabular}




\section{Discussion}

The diagnosis of primary headache is fundamentally clinical, in that there is no specific diagnostic test or biological marker with pathognomonic value available at present. The negative results obtained between attacks in the general and neurological examinations is a constant in primary headaches, and together with the clinical history, allows the physician to reach a diagnosis in most of the cases. Different authors $[8,9]$ have demonstrated the validity of the semi-structured questionnaire, which is able to examine accurately the medical history of the patient, avoiding the subjective aspects of the enquiry. Furthermore, recently a self-administered questionnaire was evaluated for the diagnosis of migraine in primary care [10]. Since 1988, the IHS [4] has utilised a classification system that defines headaches on the basis of mainly anamnestic-clinical operative criteria; this classification was recently updated [1].

Osmophobia is often referred to during a migraine attack in association with phono- and photophobia. In the appendix of the second edition of the International Headache Society Classification [1], osmophobia has been proposed in the associated symptoms category of the criteria for the diagnosis of migraine. This symptom has not been studied, however, in relation to the forms of primary headache.

In our study of 775 patients, $42 \%$ with migraine and $7 \%$ with ECH reported osmophobia during the attacks; none among the ETTH and the OPH patients suffered this symptom. Interestingly, among osmophobic ECH patients, 2 of 3 patients also reported nausea, phono- and photophobia during the attacks; osmophobia seems therefore to be present in the forms of ECH sharing neurovegetative aspects with migraine.

\section{Conclusions}

Osmophobia was not referred to by the patients with ETTH and OPH headaches; thus it can be considered a peculiar symptom of migraine in respect to these forms of primary headaches. Moreover, from this limited series it seems to be a good discriminant also for $\mathrm{OPH}$, and for $\mathrm{ECH}$ patients not sharing neurovegetative symptoms with migraine. On the basis of these data, osmophobia should be considered a good candidate as a new criterion for the diagnosis of migraine.

\section{References}

1. Headache Classification Subcommittee of the International Headache Society (2004) The International Classification of Headache Disorders, 2nd edn. Cephalalgia 24[Suppl 1]:9-160

2. Ad Hoc Committee of the Italian Society for the Study of Headaches (2001) Migraine diagnosis. J Headache Pain 2:111-116

3. Pryse-Phillips WE, Dodick DW, Edmeads JG, Gawel MJ, Nelson RF, Purdy RA, Robinson G, Stirling D, Worthington I (1997) Guidelines for the diagnosis and management of migraine in clinical practice. Canadian Headache Society. Can Med Assoc J 156:1273-1287
4. Headache Classification Committee of the International Headache Society (1988) Classification and diagnostic criteria for headache disorders, cranial neuralgias and facial pain. Cephalalgia 8[Suppl 7]:1-96

5. Blau JN, Solomon F (1985) Smell and other sensory disturbances in migraine. J Neurol 232:275-276

6. Kelman L (2004) The place of osmophobia and taste abnormalities in migraine classification: a tertiary care study of 1237 patients. Cephalalgia 24:940-946

7. Morillo LE, Alarcon F, Aranaga N et al (2005) Latin American Migraine Study Group. Clinical characteristics and patterns of medication use of migraineurs in Latin America from 12 cities in 6 countries. Headache 45:118-126
8. Iversen HK, Langemark M, Andersson PG et al (1990) Clinical characteristics of migraine and episodic tension-type headache in relation to old and new diagnostic criteria. Headache 30:514-519

9. Tietjen GE (1996) The primary headache disorders - apple sauce or fruit cocktail? Cephalalgia 16:223

10. Lipton RB, Dodick D, Sadovsky R et al (2003) A self-administered screener for migraine in primary care: the ID Migraine validation study. Neurology 61:375-382 